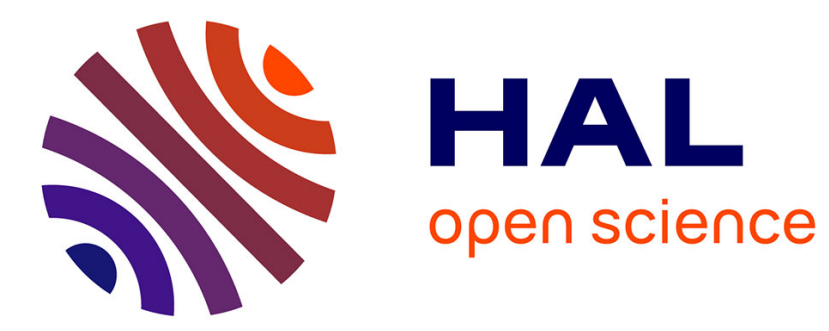

\title{
Quelques interrogations concernant la politique de rechargement pratiquée sur la flèche de galets des bas-champs de Cayeux (Somme)
}

\author{
Franck Dolique
}

\section{- To cite this version:}

Franck Dolique. Quelques interrogations concernant la politique de rechargement pratiquée sur la flèche de galets des bas-champs de Cayeux (Somme). Hommes et Terres du Nord, 1999, 2 (1), pp.107112. 10.3406/htn.1999.2675 . hal-02542769

\section{HAL Id: hal-02542769 \\ https://hal.science/hal-02542769}

Submitted on 8 Feb 2021

HAL is a multi-disciplinary open access archive for the deposit and dissemination of scientific research documents, whether they are published or not. The documents may come from teaching and research institutions in France or abroad, or from public or private research centers.
L'archive ouverte pluridisciplinaire HAL, est destinée au dépôt et à la diffusion de documents scientifiques de niveau recherche, publiés ou non, émanant des établissements d'enseignement et de recherche français ou étrangers, des laboratoires publics ou privés.

\section{(c)(1)}

Distributed under a Creative Commons Attribution| 4.0 International License 


\title{
Quelques interrogations concernant la politique de rechargement
} pratiquée sur la flèche de galets des bas-champs de Cayeux (Somme)

\author{
Monsieur Franck Dolique
}

\section{Citer ce document / Cite this document :}

Dolique Franck. Quelques interrogations concernant la politique de rechargement pratiquée sur la flèche de galets des baschamps de Cayeux (Somme). In: Hommes et Terres du Nord, 1999/2. Etudes picardes. Hommage à Pierre Limouzin. pp. 107112;

doi : https://doi.org/10.3406/htn.1999.2675

https://www.persee.fr/doc/htn_0018-439x_1999_num_2_1_2675

Fichier pdf généré le 08/05/2018 


\begin{abstract}
Defense policy envisaged in order to counter the threat of marine erosion of the proximal sector of the gravel spit of Bas-Champs de Cayeux, in Picardy, essentially involves an initial nourishment project, followed by periodic recharge to the tune of a total of $400,000 \mathrm{~m} 3$ over 5 years. Various questions arise concerning the pertinence of this policy. The aim here is not to call into question the validity of artificial nouriishment, which has proved its efficacy in numerous case around the world, on the condition of ready access to recharge material. The morphodynamic réactions of gravel nourishment are still rather poorly known. This article proposes a framework of reflection on this aspect using the gravel spit of Bas-Champs de Cayeux as an experimental example.
\end{abstract}

\title{
Résumé
}

La politique de défense contre la mer envisagée pour limiter le démaigrissement de la section proximale de la flèche de galets des Bas-Champs de Cayeux repose sur une alimentation initiale accompagnée de rechargements ponctuels pour un volume total de $400000 \mathrm{~m} 3$ sur 5 ans. De nombreuses questions se posent concernant cette politique de gestion. II n'est pas de notre volonté de remettre en question le principe même de la méthode qui, au vu de ce qui est pratiqué dans le monde, est la meilleure dans le cadre de réductions des réserves sédimentaires utilisables, encore faut-il trouver une ressource de remplacement en quantité suffisante. Les réactions morphodynamiques des rechargements de galets sont encore mal connues. Cet article jette les bases d'une réflexion sur cet aspect en utilisant le cordon des Bas-Champs comme un laboratoire d'expérimentation. 


\title{
Quelques interrogations concernant la politique de rechargement pratiquée sur la flèche de galets des Bas-Champs de Cayeux (Somme)
}

\author{
Franck DOLIQUE \\ Université du littoral \\ Laboratoire de géomorphologie dynamique et \\ aménagement des littoraux \\ JE 2208 MENESR \\ 2, Chaussée des Darses \\ 59140 DUNKERQUE \\ E-mail : dolique@univ-littoral.fr
}

\begin{abstract}
Résumé
La politique de défense contre la mer envisagée pour limiter le démaigrissement de la section proximale de la flèche de galets des Bas-Champs de Cayeux repose sur une alimentation initiale accompagnée de rechargements ponctuels pour un volume total de $400000 \mathrm{~m} 3$ sur 5 ans. De nombreuses questions se posent concernant cette politique de gestion. II n'est pas de notre volonté de remettre en question le principe même de la méthode qui, au vu de ce qui est pratiqué dans le monde, est la meilleure dans le cadre de réductions des réserves sédimentaires utilisables, encore faut-il trouver une ressource de remplacement en quantité suffisante. Les réactions morphodynamiques des rechargements de galets sont encore mal connues. Cet article jette les bases d'une réflexion sur cet aspect en utilisant le cordon des Bas-Champs comme un laboratoire d'expérimentation.
\end{abstract}

Mots-clés : rechargement, gestion littorale, galets, morphodynamique, Bas-Champs de Cayeux, Picardie.

\begin{abstract}
Defense policy envisaged in order to counter the threat of marine erosion of the proximal sector of the gravel spit of Bas-Champs de Cayeux, in Picardy, essentially involves an initial nourishment project, followed by periodic recharge to the tune of a total of $400,000 \mathrm{~m} 3$ over 5 years. Various questions arise concerning the pertinence of this policy. The aim here is not to call into question the validity of artificial nouriishment, which has proved its efficacy in numerous case around the world, on the condition of ready access to recharge material. The morphodynamic réactions of gravel nourishment are still rather poorly known. This article proposes a framework of reflection on this aspect using the gravel spit of Bas-Champs de Cayeux as an experimental example.
\end{abstract}

Key-words : beach nourishment, coastal planning, gravel, morphodynamics, Bas-Champs de Cayeux, Picardy.

politiques. Les uns préconisent un abandon des BasChamps et un "laisser-faire " par les forces naturelles, d'autres pensent qu'il est nécessaire de maintenir le trait de côte sur son tracé actuel afin d'avoir à éviter d'exposer la ville de Cayeux à des problèmes d'érosion encore plus inquiétants.

Par cet article, l'auteur soulève un certain nombre de questions (individualisées ici par paragraphes) sur la nouvelle orientation de défense contre la mer mais il ne cherche nullement à polémiquer sur un aménagement qui est, certes, contestable mais également nécessaire pour la ville de Cayeux.

\section{Rappel de la situation}

La flèche de galets des Bas-Champs de Cayeux est une importante forme sédimentaire de 16 kilomètres de long, large de 100 à $600 \mathrm{~m}$ et d'une altitude de $+8 \mathrm{~m}$ IGN. (Fig. 1). Elle sépare la Manche de la plaine maritime basse formant les Bas-Champs, 
Figure 1. Situation et rechargements

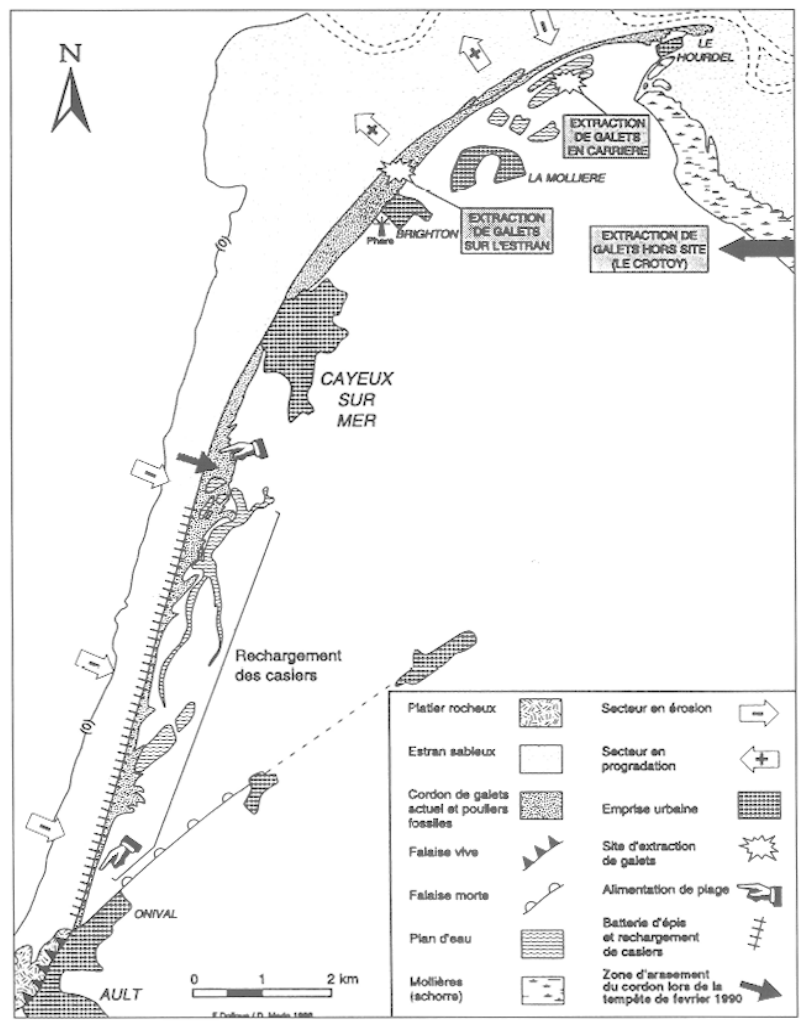

en constituant une véritable barrière littorale de protection contre les assauts de la mer car cette plaine maritime, d'une altitude moyenne de +4 à $+5 \mathrm{~m}$ IGN, soit +9 à $+10 \mathrm{~m} \mathrm{CM}$, se situe à 2 ou 3 mètres en dessous du niveau des plus hautes marées, ce qui représente un risque constant d'inondation, surtout lors des surcotes de tempêtes.

Depuis plusieurs siècles, ce cordon de galets subit des démaigrissements qui peuvent être préjudiciables et qui se traduisent par un recul du trait de la flèche au niveau de sa racine (BRIQUET, 1930 ; DALLERY, 1955 ; DOLIQUE, 1998). Cette raréfaction de la ressource sédimentaire est liée principalement aux barrages que représentent les jetées portuaires (Dieppe ou le Tréport par exemple) et qui stoppent le transit naturel des galets depuis les falaises de Normandie (DOLIQUE, 1991 ; COSTA, 1995) ; ainsi que par les prélèvements massifs, pratiqués pour des raisons commerciales sur la flèche des BasChamps et en Normandie depuis le XIXème siècle (MOREL, 1995). Cette situation a motivé la mise en place, à partir de 1966, d'une politique de défense contre la mer. Jusqu'à cette date, les initiatives de défense restaient limitées car l'ampleur du problème et son coût étaient trop importants pour la population locale. En 1966, l'Association Syndicale Autorisée des Bas-Champs de la Somme (l'ASABS) a réussi à convaincre l'Etat de la gravité de la situation pour les populations locales et a obtenu des crédits importants. A la suite de nombreuses études, les décideurs se sont orientés vers la construction de séries d'épis. Au vu de la forte dynamique des galets dans cette région, il s'est avéré que cet aménagement ne serait pas viable sans rechargements et entretien réguliers. Au total, ce sont 55 épis de 90 mètres de long, espacés de 90 mètres, qui ont été construits sur un linéaire de 4,5 kilomètres pour une dépense financière estimée en 1989 à 45 millions de francs (DDE, 1991). Entre le 27 février et le 3 mars 1990, une tempête violente, générant des vents d'ouest de $155 \mathrm{~km} / \mathrm{h}$ a fait rage sur les côtes de la Manche orientale. La marée haute, à fort coefficient (104), a été surcotée par les vents, provoquant une brèche d'un peu plus d'un kilomètre en aval-dérive de la batterie d'épis. La mer a envahi les Bas-Champs sur une surface de 3000 hectares environ provoquant des pertes de bétail, des stérilisations de sols ainsi que des destructions d'équipements agricoles et des huttes de chasse. Cette catastrophe marqua profondément les esprits des populations locales et par conséquent des décideurs. La responsabilité, pourtant évidente, de la batterie d'épis dans cette inondation ne fut pas explicitement reconnue. Les carriers furent, aux yeux de la population, tenus responsables de l'amenuisement du stock sédimentaire malgré l'interdiction d'exploitation sur la zone Ault-Onival - Cayeux établie depuis 1983. Quelles que soient les responsabilités, il fut décidé d'entamer une réflexion concernant la politique à adopter pour la lutte contre l'érosion. Plusieurs études furent lancées afin de déterminer un modèle d'aménagement adapté. Diverses solutions furent proposées entre un abandon du trait de côte, une artificialisation totale par enrochements et une gamme de solutions intermédiaires. L'idée d'un modèle dynamique "souple "fondé sur une alimentation de plage initiale et des rechargements ponctuels, méthode de plus en plus usitée en matière de défense littorale, fut acceptée dans son principe mais il fut également décidé de poursuivre et de renforcer les structures déjà existantes. S'il existe effectivement une volonté de pratiquer une méthode " douce " d'aménagement, elle ne s'appliquera que dans le cadre du renforcement d'une méthode " dure ". Ce cas est suffisamment original pour être souligné.

\section{Les nouvelles orientations de défense contre la mer}

La nouvelle politique d'aménagement du cordon littoral, adoptée en 1995, se fonde sur une alimentation et des rechargements massifs. Le modèle retenu peut se résumer comme suit :

- on procède sur le cordon à une alimentation initiale de galets pour un volume de 200000 à $300000 \mathrm{~m}^{3}$,

- par mesure d'entretien, des rechargements réguliers des casiers pour un volume de $20000 \mathrm{~m} 3 / \mathrm{an}$ environ sont effectués,

- il est ensuite nécessaire de redresser l'anse d'érosion en aval-dérive de la batterie d'épis par des rechargements et éventuellement par la mise en place d'un épaulement en arrière du cordon.

Ces rechargements nécessitent un transport des galets par camions, ce qui représente 30 camions mobilisés contenant chacun 20 à 24 tonnes de galets à raison d'un camion toutes les trois minutes sur une base de 50 à $80 \mathrm{~F}$ le mètre cube. Une soixantaine de personnes sont amenées à travailler 
en permanence sur ce chantier pharaonique prévu pour durer quatre ans.

Ces mesures de rechargement s'accompagnent d'un programme de renforcement de la politique précédente avec :

- une restauration des 55 épis de palplanches existants,

- une extension de la batterie d'épis jusqu'à Cayeux, secteur pourtant dynamiquement stable actuellement ( 35 épis supplémentaires sur $3 \mathrm{~km}$ ).

Au moment où cet article a été rédigé (fin 1998), les épis existants ont déjà été restaurés et la mise en place des 35 épis supplémentaires, selon une technique de préfabrication faite sur le site, vient de s'achever. Ces 35 épis représentent 3150 mètres de linéaire, soit l'équivalent du linéaire de côte traité. Chacun de ces épis est lons de $90 \mathrm{~m}$ et les casiers sont larges de $90 \mathrm{~m}$. Les palplanches sont enfoncées par un vibreur et coiffées par un " chapeau " de béton devant assurer la longévité des épis contre les chocs des galets et empêcher l'oxydation du métal. Le béton, d'une couleur proche de celle des galets, a pour ambition également de faire oublier les épis en les " intégrant " au paysage, ce qui semble difficile ! Globalement, le coût de ce programme d'aménagement, initialement prévu à 125 millions de Francs, a été révisé aux alentours de 150 millions de Francs répartis entre l'Etat, la Région, le Département et la Communauté Européenne (soit un coût par habitant de 37500 Francs). II est fort probable que la facture finale dépassera cette estimation, ce qui ferait des Bas-Champs l'un des littoraux les plus coûteux de France et même d'Europe.

\section{Des interrogations concernant la ressource}

L'aménagement retenu est fondé sur une alimentation initiale de plage et de rechargements. L'apport initial est estimé entre 200000 et $300000 \mathrm{~m} 3$ et les rechargements annuels seront de $20000 \mathrm{~m} 3 / \mathrm{an}$ environ. Où trouver un tel volume de matériaux, d'autant qu'il semble fondamental que les galets soient de bonne qualité, c'est à dire résistants et d'une granulométrie supérieure à $40 \mathrm{~mm}$.

\section{Vers un conflit d'usage avec les carriers?}

On s'oriente tout d'abord vers les carrières déjà exploitées situées en arrière du cordon littoral, au nord de Cayeux (carrière du Hourdel: 70 hectares) ainsi que vers celles situées en dehors du site, au niveau de St Firmin-les-Crotoy, Rue, Waben, qui exploitent les galets sur des pouliers internes pléistocènes. Un partenariat avec les exploitants est conclu sous la forme d'un partage de la ressource : les carriers se réservant les galets ronds riches en silice, la DDE récupérant les galets oxydés sans valeur commerciale, ayant de préférence une granulométrie supérieure à $40 \mathrm{~mm}$. Or, dans un contexte actuel d'épuisement de la ressource, il semble que les stocks de carrières ne seront pas suffisants, à l'image de la carrière du Hourdel dont on annonce un arrêt d'exploitation programmé et une reconversion du plan d'eau vers une activité de "nature " ou de loisirs. Cette cohabitation entre les carriers dont la vocation est l'exploitation des galets à des fins industrielles et les gestionnaires qui ont en charge la défense du trait de côte, et qui exploitent la ressource à partir des mêmes lieux, ne peut que devenir problématique à long terme. II faut donc rechercher des ressources complémentaires ailleurs. On a évoqué la possibilité de puiser sur les crochets fossiles situés en arrière du cordon. Cette solution n'est pas souhaitable car ces cordons jouent un rôle d'épaulement pour le cordon vif. D'autres solutions, dont la viabilité est liée aux coûts et aux difficultés techniques, ont été envisagées et doivent être étudiées de près.

\section{Vers une ressource externe coûteuse?}

- Tout d'abord, il a été évoqué la possibilité de recourir à des stocks sous-marins situés au large de Dieppe. Cette solution pose le problème d'un coût d'exploitation exorbitant pour une ressource composée de sédiments très disparates avec une courbe granulométrique étalée montrant la faible teneur en galets de granulométrie significative. II s'agit, en fait, essentiellement de graviers. Cette méthode est toutefois utilisée avec un certain succès en Angleterre (Seaford, Sussex) mais là, les stocks sont de nature différente et proches de la plage à recharger, d'où une diminution du coût de transport. D'autre part, ce type d'extraction peut être néfaste pour l'équilibre sédimentaire de l'avant-plage. Les conséquences de l'extraction de granulats marins sont encore mal connues mais des expériences (ARISTAGHES, 1987) montrent qu'une souille, en se comblant, peut augmenter la pente de l'avant-plage ce qui amplifiera la puissance du déferlement. Ce n'est qu'un des aspects physiques inconnus d'une extraction en mer. L'expérience anglaise et les débuts de concertation en France montrent qu'une telle pratique pourrait très vite ouvrir un conflit d'usage avec les pêcheurs (travaux table ronde de la réunion " arc-Manche ", Rouen, 16 et 17 octobre 1997).

- Il est envisagé un rechargement à partir de galets importés depuis les plages anglaises par cargos qui déchargeraient au Tréport. Cette solution semble irréaliste du fait d'un coût exorbitant (extraction + transport). La différence de qualité semble être un argument négatif, lui aussi, si on prend en considération la granulométrie plus fine du galet anglais, ainsi que sa couleur rouille caractéristique qui risque de rompre l'harmonie visuelle du cordon (d'ailleurs, peut-on encore parler d'harmonie visuelle si on prend en considération la batterie d'épis ?). Enfin, on peut se demander si le contexte morphosédimentaire, à tendance érosive en Angleterre comme en France, peut permettre aux Britanniques de laisser partir leur ressource de galets?

\section{Quelle adaptation morphodynamique pour les rechargements?}

L'évolution dynamique du rechargement : conjectures.

L'une des interrogations les plus intéressantes concernant la politique de rechargement est de 
savoir quelle sera la réaction morphodynamique des apports, les rechargements peuvent-ils réellement se substituer aux galets déjà en place et la plage vat'elle évoluer comme on l'attend. II est difficile de répondre à ces questions sans avoir réalisé auparavant un grand nombre d'expérimentations sur le terrain. Des études, menées dans le cadre d'une thèse de doctorat permettent de tirer de premiers enseignements (DOLIQUE, 1998).

La littérature géomorphologique offre un certain nombre d'exemples de réactions morphodynamiques par rapport aux rechargements. Des rechargements effectués à Gelendzhik Bay, sur les bords de la Mer Noire, donnent des résultats assez satisfaisants même si on note, deux ans après les premiers rechargements, un abaissement de la base du profil transversal (KOS'YAN et al., 1994). Dans la même région, un autre exemple nous est fourni par KIKNADZE et al. (1990) et par CABANNE et MIOSSEC (1994). Sur cette côte géorgienne de la Mer Noire, les rechargements ont donné des résultats satisfaisants par rapport aux méthodes de défense statiques jusqu'ici employées et qui ont montré leurs limites. Ici, le profil est invariable. Ces deux cas se situent en milieu microtidal et on peut imaginer des résultats différents en milieu macrotidal. Selon KIKNADZE, les forts marnages ne peuvent que donner de meilleurs résultats par rapport aux méthodes adoptées sur la Mer Noire (CABANNE et MIOSSEC, 1994). On peut douter de la stabilité du rechargement en ce qui concerne le littoral macrotidal des Bas-Champs puisque de forts taux d'accrétion mesurés au niveau de La Mollière démontrent la forte mobilité du sédiment dans ce contexte de haute énergie (DOLIQUE et ANTHONY, 1998). En NouvelleZélande, KIRK (1992) signale des pertes vers l'avantcôte de l'ordre de 4 à $7 \%$ alors que COHEN (1996) fait état du même problème avec une disparition des rechargements vers des micro-canyons pour la plage de Nice. Ce problème ne se pose pas sur les Bas-Champs dans la mesure où la présence d'une plate-forme sableuse annihile toute descente gravitaire. NICHOLLS (1990) pense qu'il est important de faire une distinction entre les plages à haut de plage graveleux et à avant-plage graveleuse ( $\mathrm{Sh} / \mathrm{Sh}$ ) et les plages à haut de plage graveleux et à avant-plage sableuse $(\mathrm{Sh} / \mathrm{Sa})$. Les grèves (Sh/Sh) voient leur rechargement glisser régulièrement vers les fonds par la pente alors que les plages ( $\mathrm{Sh} / \mathrm{Sa}$ ), comme pour le cordon des Bas-Champs, conservent leur rechargement du fait de la très faible pente de la plate-forme sableuse. On peut donc penser que la redistribution du rechargement sera, pour les BasChamps, essentiellement longitudinale (longshore) et non transversale (cross-shore). Les exemples de réussite en milieu macrotidal sont assez nombreux en Angleterre et notamment à Dungeness (MUIRWOOD, 1970) et dans le Sussex (FOXLEY et SHAVE, 1983). POWELL (1989), pense que les rechargements de galets constituent la meilleure solution de défense contre la mer pour les plages de galets en s'appuyant sur les résultats des plages de Hayling (500 000 m3 en 1985) et Seaford (1500 000 m3 de granulats marins dragués au large, en 1987). Pour le cordon des Bas-Champs, quelques remarques per- mettent d'anticiper les principales réactions morphodynamiques.

On peut supposer que les casiers de la batterie d'épis seront bien remplis par les rechargements, ce qui laissera échapper un volume important par débordement vers le haut d'ouvrage. Toutefois, cela ne sera certainement pas suffisant pour assurer la stabilité de la fragile plage de Cayeux-sur-Mer, située en aval-dérive de la batterie, qui montrait jusque-là des phases alternatives de démaigrissement et d'engraissement.

\section{Le recyclage des galets est-il viable longtemps?}

Depuis 1921, il se développe une importante accumulation en cordons successifs au droit de La Mollière (DOLIQUE, 1997). Ce stock, déjà en partie exploité par des entreprises autorisées à ramasser des galets sur le Domaine Public Maritime, sert actuellement de réserve pour les rechargements d'entretien réalisés par la DDE. On compte ainsi sur un recyclage des galets puisqu'à terme, les galets injectés au sud de la flèche viendront se déposer de nouveau au nord, sur cette même zone d'extraction. Mais là aussi, on peut se poser un certain nombre de questions sur la méthode. Tout d'abord, il convient d'être prudent avec les tonnages prélevés car les flux nord-sud seront plus élevés et plus rapides (camions) que les flux sud-nord, dont une partie du volume sera retenue par les épis et le transport assuré par la dérive littorale. On risque donc de déséquilibrer le bilan de ce recyclage en imposant des départs plus importants depuis la zone d'extraction que ne seront les arrivées. Cela risque de déséquilibrer à terme ce milieu naturel séomorphologique et écologique d'un intérêt scientifique et pédagogique fondamental composé de flèches successives régulièrement fossilisées, entrecoupées de petites dépressions marécageuses comportant plusieurs stades de colonisation végétale. D'autre part, si on prend en considération l'usure des galets par frottements et attritions, (des esquilles de silex représentent $10 \%$ des sables intertidaux : BEAUCHAMP, 1994), une usure rapide des galets supérieurs à $40 \mathrm{~mm}$ devrait se produire, raréfiant le stock de cette classe granulométrique essentielle au rechargement mais plus rare que les classes inférieures.

\section{Attention à la rupture du profil.}

La part de la fraction sableuse interstitielle peut jouer un rôle très important sur la réaction morphodynamique du rechargement mais ce rôle n'est pas encore totalement défini. II en va de même pour un rechargement qui aurait une granulométrie différente de celle du sédiment en place. Le granulat de carrière (utilisé pour les Bas-Champs), tout comme le granulat marin, possède une importante part de fraction sableuse. Celle-ci, une fois saturée en eau, renforce le caractère réfléchissant d'une grève vis-àvis des houles mais peut aussi stabiliser les galets en jouant le rôle d'une matrice piégeante. Ici, les matériaux de carrière doivent en principe être filtrés (le seront-ils toujours ?) pour sélectionner les granulo- 
Figure 2. Profils de rechargement: comparaison (Onival Nord)

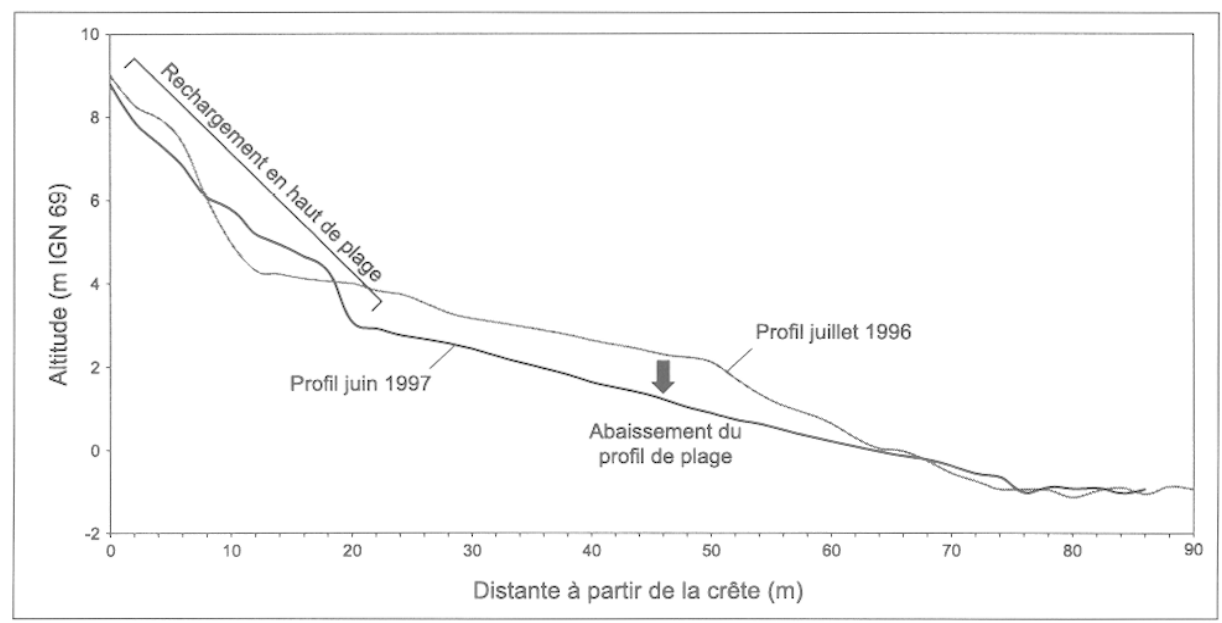

des microfracturations par cryoclasties et cryoturbations lors de la dernière glaciation. II m'a semblé nécessaire de réaliser des tests de résistance mécanique afin de tenter de confirmer ou d'infirmer cette hypothèse. Ces tests ont été effectués sur deux échantillons de galets. Le premier échantillon était constitué de galets prélevés sur le cordon et nommé " MAR " pour " galet de mer ". Le second échantillon a été prélevé sur un site de rechargement et

métries supérieures à $40 \mathrm{~mm}$. Ainsi, une bonne part des sables doit être éliminée. L'absence de sable au sein du rechargement va certainement faciliter la mise en circulation du volume injecté. Par ailleurs, une granulométrie plus grossière du rechargement (facteur de stabilité par le poids plus important du galet), risque de renforcer le caractère réfléchissant de la plage (POWELL, 1994) et former à terme une rupture de pente lorsque le rechargement est situé en haut de plage comme c'est le cas pour les BasChamps. Cette rupture de pente peut avoir un rôle fondamental pour le comportement de la plage. En effet, le rechargement de forte granulométrie, placé en haut de cordon pour être progressivement redistribué par le déferlement, va avoir une pente initiale plus raide que la pente du profil naturel de plage. Un levé topographique a été réalisé au tachéomètre infra-rouge sur un tronçon de plage au nord d'Onival, en juillet 1996 (Fig. 2) et montre parfaitement cette rupture dans le profil. Le même travail fut réalisé au même endroit en juin 1997. La comparaison des deux profils topographiques montre un net abaissement de la section non rechargée du cordon (presque 1 mètre), lié à la forte pente de la section rechargée. La présence de cette pente raide en haut de cordon a joué ici le rôle d'un mur réfléchissant l'énergie des vagues en amplifiant la force de la nappe de retrait (backwash) qui a mobilisé les galets et les a fait migrer (Fig. 2). Un rechargement de haut de cordon peut donc être très dommageable pour le bas de cordon qui peut subir une érosion, si on ne prend pas soin d'uniformiser la pente du profil.

La qualité du matériau de rechargement est également importante. C'est pour cela qu'il est apparu nécessaire d'effectuer des tests de résistance mécanique sur des échantillons.

\section{Expérimentations concernant la résistance mécanique des matériaux}

Des observations visuelles font état d'une moindre résistance des galets de carrière par rapport aux galets de la flèche. En effet, les gisements de galets exploités dans la région du Crotoy sont des bancs fossiles pléistocènes dont les galets auraient subi nommé "CAR " pour " galet de carrière ". La différence de couleur et d'aspect est suffisamment nette pour éviter toute confusion entre les deux types de granulats. Le laboratoire régional des ponts et chaussées de Lille s'est chargé de l'expérimentation de type "Los Angeles ". Cette expérimentation consiste à mesurer la résistance de granulats aux chocs en plaçant l'échantillon testé dans un tambour rotatif au sein duquel des boulets d'acier jouent le rôle de broyeur. Les échantillons, d'un poids approximatif de $15 \mathrm{~kg}$ chacun, ont été décomposés en classes granulaires de 10-25 mm, 25-31 mm, 31,5-40 mm et 40-50 mm, en essayant de tenir une proportion de $25 \%$ par classes. Après broyage, on pèse les résidus d'éléments inférieurs à $1,6 \mathrm{~mm}$. Le résultat s'exprime par une valeur numérique $L A=(m / M) .100$ où $m$ est la masse des éléments inférieurs à $1,6 \mathrm{~mm}$; $M$ est la masse du matériau soumis à l'essai. Donc, plus la valeur de LA est faible, plus l'échantillon est résistant. Les résultats obtenus sont synthétisés dans la figure 3. Ces résultats semblent démontrer le contraire de ce qui a été qualitativement supposé. Les indices LA montrent que les galets de carrière ont fourni moins de résidus fins. Cela voudrait dire que les galets de rechargement de la digue des Bas-Champs offrent une résistance supérieure aux galets déjà en place, ce qui est très intéressant dans le cadre d'un rechargement. II faut toutefois prendre ces résultats avec prudence pour au moins deux raisons :

- L'écart entre les deux valeurs de LA est faible et peut ne pas être suffisamment représentatif, d'autant que cette expérience devra être renouvelée avec un plus grand nombre d'échantillons.

- Les chocs sur les galets sont très violents et ne sont pas représentatifs d'une réalité de terrain où les galets subissent une attrition et des chocs légers,

Figure 3. Résultats des essais LOS ANGELES (LA)

\begin{tabular}{|l|c|c|}
\cline { 2 - 3 } \multicolumn{1}{|c|}{} & Echantillon cordon (MAR) & Echantillon carrière (CAR) \\
\hline 1er essai & 30,3 & 25,9 \\
\hline 2ème essai & 31,4 & 26,7 \\
\hline
\end{tabular}




\section{CONCLUSION}

L'inondation de février 1990 a fait prendre conscience qu'un changement dans la politique de défense contre la mer devait s'imposer. Les nouvelles orientations se sont dirigées vers des rechargements massifs. II se pose alors la question de la provenance d'une ressource aussi importante. En prévision d'un épuisement progressif des stocks de carrières actuellement exploitées, les aménageurs se tournent vers des solutions d'exploitation de ressources sous-marines ou de stocks en provenance de l'étranger mais ces solutions ne sont pas satisfaisantes actuellement. La réaction morphodynamique du matériau de rechargement est également un sujet d'interrogation. L'étude de profils de plaze tend à montrer que le rechargement, de granulométrie supérieure au galet en place et injecté en haut de plage, joue le rôle de "mur " réfléchissant préjudiciable pour le bas du cordon. L'analyse de fragmentation mécanique des galets semble démontrer une meilleure résistance aux chocs pour les galets de carrière, ce qui est intéressant dans le cas d'un rechargement de plage, mais il convient d'apporter toutes les réserves nécessaires à ce genre de résultats.

La pratique des rechargements de plage dans le cas d'une érosion du trait de côte est de plus en plus utilisée dans le monde car elle représente une méthode naturelle, souple et jusqu'ici assez efficace pour contrer les pénuries de sédiments exploitables. Cependant, devant les interrogations fournies par ce modèle d'aménagement tel qu'il est pratiqué sur les Bas-Champs, les perspectives de solutions alternatives restent d'actualité (ouverture à la mer), même si elles ne sont guère plus satisfaisantes à long terme, et devraient encore, à l'heure actuelle, faire l'objet de débats.

\section{BIBLIOGRAPHIE}

ARISTAGHES (C.), 1987 Influence de l'extraction des granulats marins sur l'équilibre du littoral. Rapport STCPMVN 87-1, 16p.

BEAUCHAMP (J.), 1994 Abrasion des galets et origine du sable en Baie de Somme. Travaux du département de géologie de I'Université de Picardie, t3: sédimentologie en Baie de Somme, pp 75-86.

BRIQUET (A.), 1930 Le littoral du nord de la France, évolution et morphologie. Lib. A. Colin, Paris, 438 p. +1 appendice: L'évolution du rivage du nord de la France et l'activité de l'hom$m e, 41 \mathrm{p}$.

CABANNE (C.), MIOSSEC (A.), 1994 Un exemple de protection du littoral : la côte de la Mer Noire entre Sotchi et Batoumi Cahiers Nantais, $n^{\circ}$ 41-42, pp 99-105.

COHEN (O.), 1996 Approche méthodologique à l'étude des littoraux en Méditerranée française : exemples sur la côte d'Azur. Thèse de Doctorat, Université du Littoral, Dunkerque, $212 \mathrm{p}$.

COSTA (S.), 1995 Vulnérabilité des villes côtières de HauteNormandie et de Picardie face à l'élévation du niveau marin. Hommes et Terres du Nord, 1995 1-2, pp 48-57.

D.D.E. de la Somme, 1991 Etude globale de valorisation économique et touristique des Bas-Champs, rapport de synthèse des potentialités de la situation actuelle. Rapport DDE non publié

DALLERY (F.), 1955 Les rivages de la Somme, autrefois, aujourd'hui et demain. Mémoires de la société d'émulation historique et littéraire d'Abbeville, Ed. A. et J. Picard et Cie, Paris, 307p.

DOLIQUE (F.), 1997 Modifications morphosédimentaires par influence estuarienne sur la flèche de galets des Bas-Champs de Cayeux. actes du colloque GEOPHEN, Caen, 8, 9 et 10 mai 1997.

DOLIQUE (F.), 1998 Dynamique morphosédimentaire et aménagements induits du littoral picard au sud de la baie de Somme. Thèse de doctorat, géographie physique, Université du littoral Dunkerque, $420 \mathrm{p}$.

DOLIQUE (F.) et ANTHONY (E. J.), 1998 The gravel barrier of Cayeux-sur-Mer, Picardy, France : a summary of recent morphosedimentary changes. Journal de Recherche Océanographique, 23-
4, pp 157-162.

FOXLEY (J. C.), SHAVE (K.), 1983, Beach monitoring and shingle recharge in shoreline protection. Telford Ldt, London, $\mathrm{pp} 163$ 170.

KIKNADZE (A. G.), SAKVARELIDZE (V. V.), PESHKOV (M.), GRIGORY (E. R.), 1990 Beach forming process management of the Georgian Black Sea Coast Journal of coastal reaserch, SI 6, pp 33-44.

KIRK (R. M.), 1992 Experimental beach reconstruction-renourishment on mixed sand and gravel beachs, Washdyke Lagoon, South Canterbury, New-Zealand. Coastal Engineering, 17, pp 253-277.

KOS'YAN (R.D.), KUZNETOF (S.Y.), YESIN (N.V.), 1994 Artificial gravel beach in the throat of Gelendzhik Bay. Proc. LITTORAL 94, Lisbonne, Portugal, pp 217-230.

MOREL (V.), 1995 Impacts des actions anthropiques sur les cordons de galets. Hommes et Terres du Nord, 1995 1-2, pp 58-64.

MUIR-WOOD (A. M.), 1970 Characteristics of shingle beaches : the solution to some practical problems. Proc. of the 12th coastal engineering conference, American Society of Civil engineers, New-York, pp 1059-1076

NICHOLLS (R.J.), 1990 Managing erosion problems on shingle beaches: examples from Britain. Proceedings of 3rd European workshop on coastal zones, Paralinn, Cyprus, Sept. 90, pp 35-56.

POWEL (K. A.), 1989 The dynamic response of shingle beaches to random waves. Proc. of 21 th coastal engineering conf. ASCE, New-York, pp 1763-1773.

POWEL (K. A.) 1994 The use of dissimilar sediments for beach nourishment. Proc. LITTORAL 94, Lisbon, Portugal, pp185-199. 